\title{
Design and Flight Validation of a Communication System for an Optionally Piloted Aircraft
}

\author{
Vittorio Ugo Castrillo ${ }^{1 *}$, Francesco Fusco ${ }^{2}$ and Michele Inverno ${ }^{3}$ \\ ${ }^{1,2,3}$ C.I.R.A. - Italian Aerospace Research Center, Capua, Italy, 81043 \\ ${ }^{1}$ v.castrillo@cira.it, ${ }^{2}$ f.fusco@cira.it, ${ }^{3}$ m.inverno@cira.it
}

\begin{abstract}
In order to satisfy the flight validation needs emerging from the technology developments to support the autonomous flight of "Unmanned Aerial Systems" and the remote control of "Remotely Piloted Air System", CIRA set up a multipurpose flying platform, named Flight Laboratory for Aeronautical REsearch, based on a commercial version of the P92 Echo Super aircraft manufactured by TECNAM and modified to be remotely controlled and operated as an "Optionally Piloted Aircraft". According to the OPA paradigm, flight experiments are executed with the on-board attendance of the pilot in command who can always override the telecommands received from the Ground Control Station hosting the remote pilot. In case of aircraft remote control, the datalink should have features similar to those characterizing a datalink for an UAS even though the related impact on safety is a much less important issue. This paper describes the architecture as well as the analytical and experimental behavior of a data-link system, named Communication System, used to support the "line-of-sight" communication between the flying platform and the ground control station aimed at transferring telemetry and housekeeping data, on-board video, telecommands, configuration and services data.
\end{abstract}

Keywords: Datalink, Communication System, Telecommands, Telemetry, Unmanned Aerial Systems, Remotely Piloted Air System, Optionally Piloted Aircraft

\section{Introduction}

The flight validation of technologies for UASs (Unmanned Air Systems) or RPAS (Remotely Piloted Air System) with high Technology Readiness Level (TRL) is usually a costly and risky activity. In order to satisfy flight validation needs for RPAS enabling technologies, CIRA (Italian Aerospace Research Center) set up a multipurpose flying platform named Flight Laboratory for Aeronautical REsearch (FLARE) [1][2][3], exploiting the possibilities offered by the Optionally Piloted Aircraft (OPA) paradigm. FLARE is based on a commercial version of the P92 Echo Super aircraft produced by TECNAM and modified to integrate proprietary experimental set-ups.

According to FAA (Federal Aviation Administration) an OPA is an aircraft integrated with UAS technologies which still retains the capability of being flown by an on-board pilot using conventional control methods [4]. It means that the aircraft flies with a "safety pilot" on-board who can engage or disengage the remote control of the aircraft. Once the remote control is engaged, the aircraft is controlled by a pilot operating in the Ground Control Station (GCS). In case of malfunction or any other hazardous situation the onboard safety pilot (i.e. PIC, pilot in command) can override the telecommands received from the GCS.

Received (March 10, 2017), Review Result (June 26, 2017), Accepted (September 14, 2017)

* Corresponding Author 
By basing on this paradigm, datalink characteristics in terms of throughput, BER (Bit Error Rate) or PER (Packet Error Rate), latency time and range are the same as the datalink of an UAS. Safety issue, in terms of system reliability and resilience to voluntary and involuntary jamming, is much less demanding as a consequence of the safety pilot presence on-board.

\section{On-board Architecture}

The COMSYS is a full duplex data-link made up of two independent channels: the first one, named Wide Band Data-Link (WBDL), is a down-link channel with a throughput programmable up to $12 \mathrm{Mbps}$; the second one, named Narrow Band Data-Link (NBDL), is an up-link channel with a throughput programmable up to $1 \mathrm{Mbps}$.

Units and devices of the two channels are integrated in the on-board and ground segments, respectively installed on the aircraft and in the GCS [8].

Down-link and up-link communication channels are asynchronous, i.e. there aren't time and/or synchronization constraints between the two channels.

COMSYS on-board segment architecture (see Figure 1) was designed with the aim at maximizing its reliability. Consequently, the transmitter and the receiver are the only active devices used to implement the WBDL and NBDL RF chains. In particular, for the NBDL channel, a receiver with low noise figure $(\sim 3.5 \mathrm{~dB})$ was selected to avoid the use of a low noise amplifier.

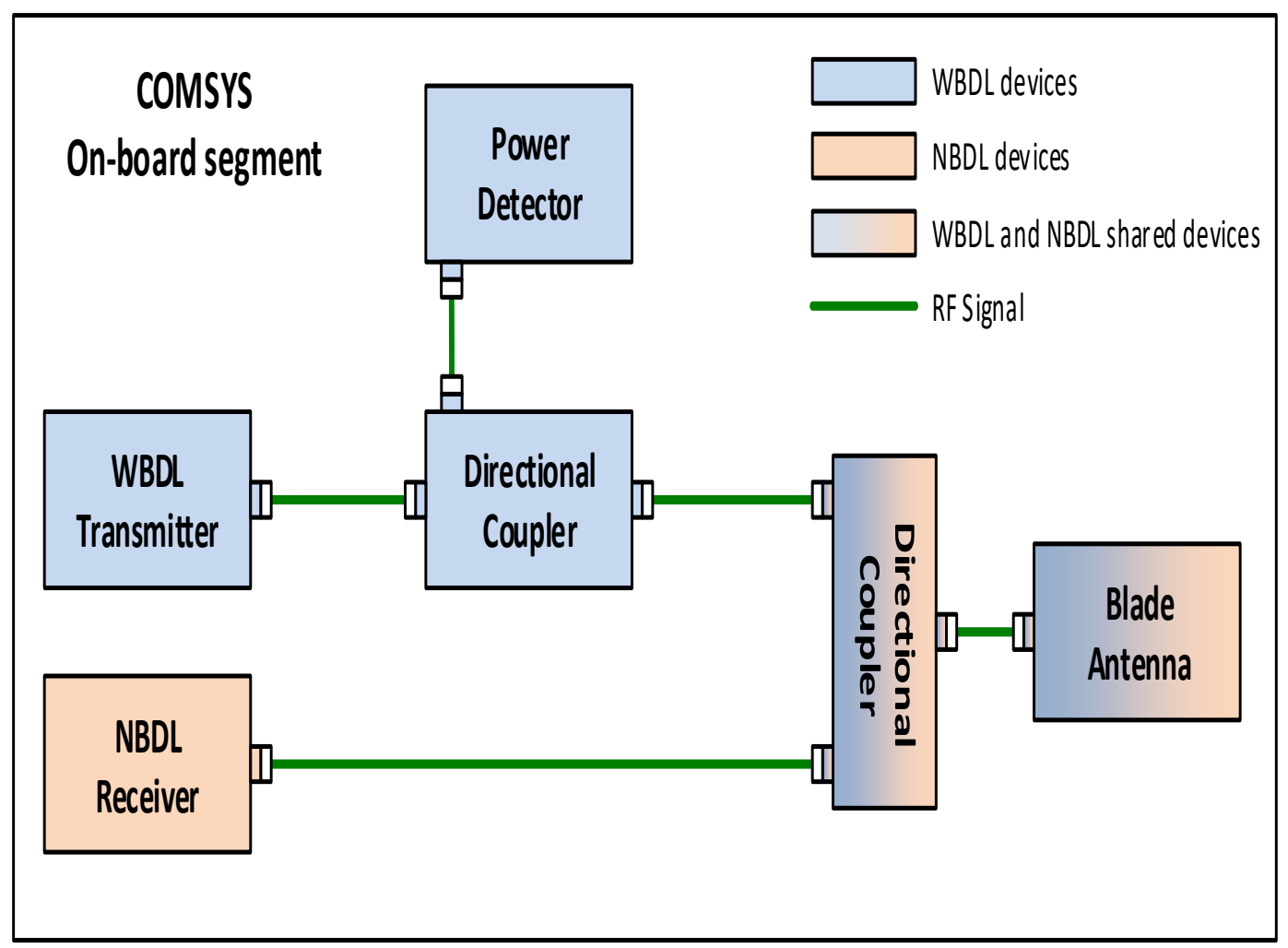

Figure 1. COMSYS on-board architecture

A signal path with a low coupling factor (-30dB) stemming from the transmitting chain allows the monitor of the RF power supplied by the transmitter. Both WBDL transmitter and NBDL receiver have built-in functions to monitor their operating parameters. 
Transmitting and receiving chains share the same blade antenna (BA), installed under the aircraft fuselage (see Figure 2), by means of a diplexer. The radiation pattern provided by the antenna after the installation was numerically evaluated using an electromagnetic simulation tool.

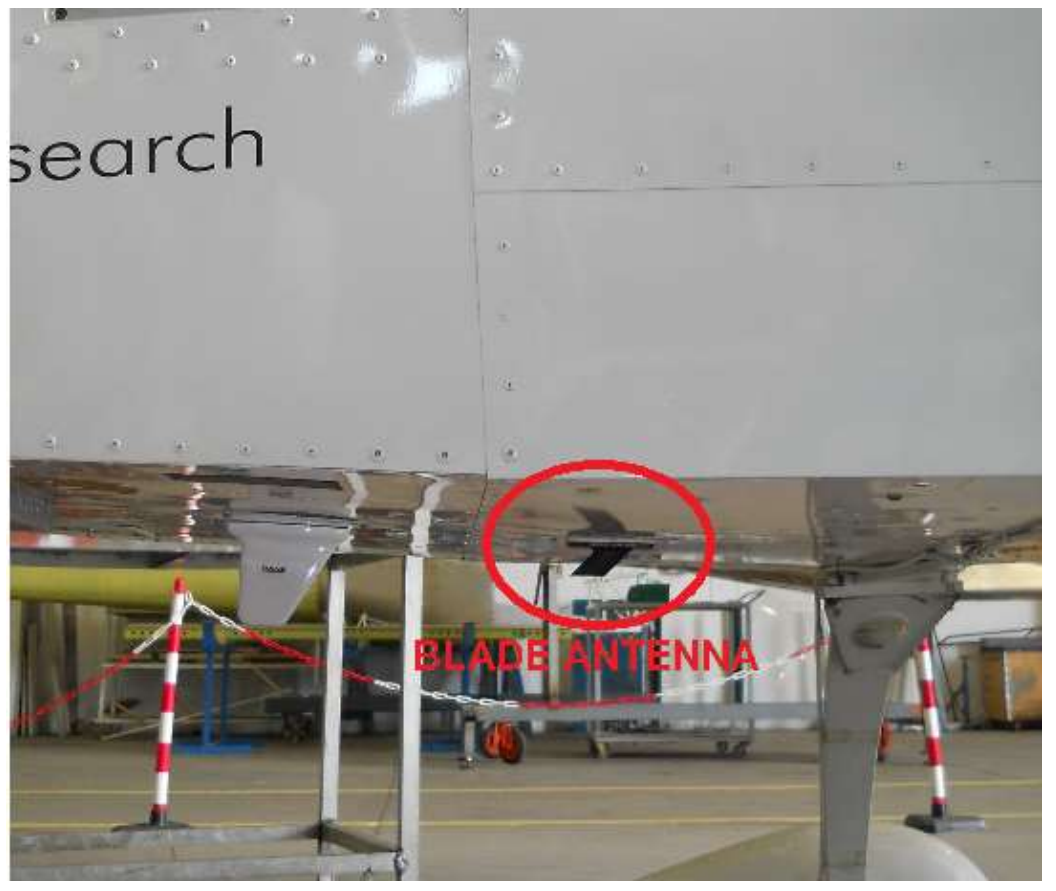

Figure 2. Blade Antenna Position

\section{Ground Architecture}

COMSYS ground segment architecture is shown in

Figure 3. By referring to the WBDL channel, the architecture shows two signal conditioning chains in reception, respectively associated to:

- a directional antenna (DA), equipped with a tracking system;

- a dedicated hemi-omni antenna (WHOA, WBDL Hemi-Omni Antenna).

The two chains are connected to the same receiver provided with two RF inputs and a pre-detection combiner operating in space diversity. Simultaneous use of the above antennas is thus possible, increasing the system availability. In such configuration, the hemi-omni antenna operates mainly when the airplane is closely passing by the ground station, i.e., when, for speed reason, the tracking executed by the directional antenna could be more difficult. The directional antenna operates instead when the airplane is far from the GCS, i.e., when the tracking of the aircraft is easier and the hemi-omni antenna cannot guarantee radio coverage because of its low gain.

Both signal conditioning chains are equipped with remotely controllable attenuators to prevent saturation or damage of the devices used in reception. Furthermore, the directional antenna can be uncoupled, by means of a RF switch, from the related conditioning chain when the distance between the aircraft and the directional antenna is so small that the related attenuator cannot provide sufficient attenuation. The attenuators system and the RF switch can be controlled manually or automatically by means of a dedicated software. In automatic control mode, attenuators and switches are operated by basing on the received RF power level and the aircraft GPS position sent to the ground station within the telemetry data frame by means of the WBDL channel. 
By referring to the NBDL channel, COMSYS architecture integrates two separate signal conditioning chains for the transmission, respectively associated to:

- the same directional antenna (DA) used for WBDL (see

- Figure 3),

- a dedicated hemi-omni antenna (NHOA, NBDL Hemi-Omni Antenna).

The two antennas are alternatively connected to the ground transmitter by means of a RF switching system made up by three RF switches. This system can be remotely controlled manually or automatically using the above mentioned software. In automatic control mode, the selection of the suitable antenna is based on the aircraft GPS position (received through the WBDL cannel), the GPS ground station position and the coverage diagrams of the two antennas.

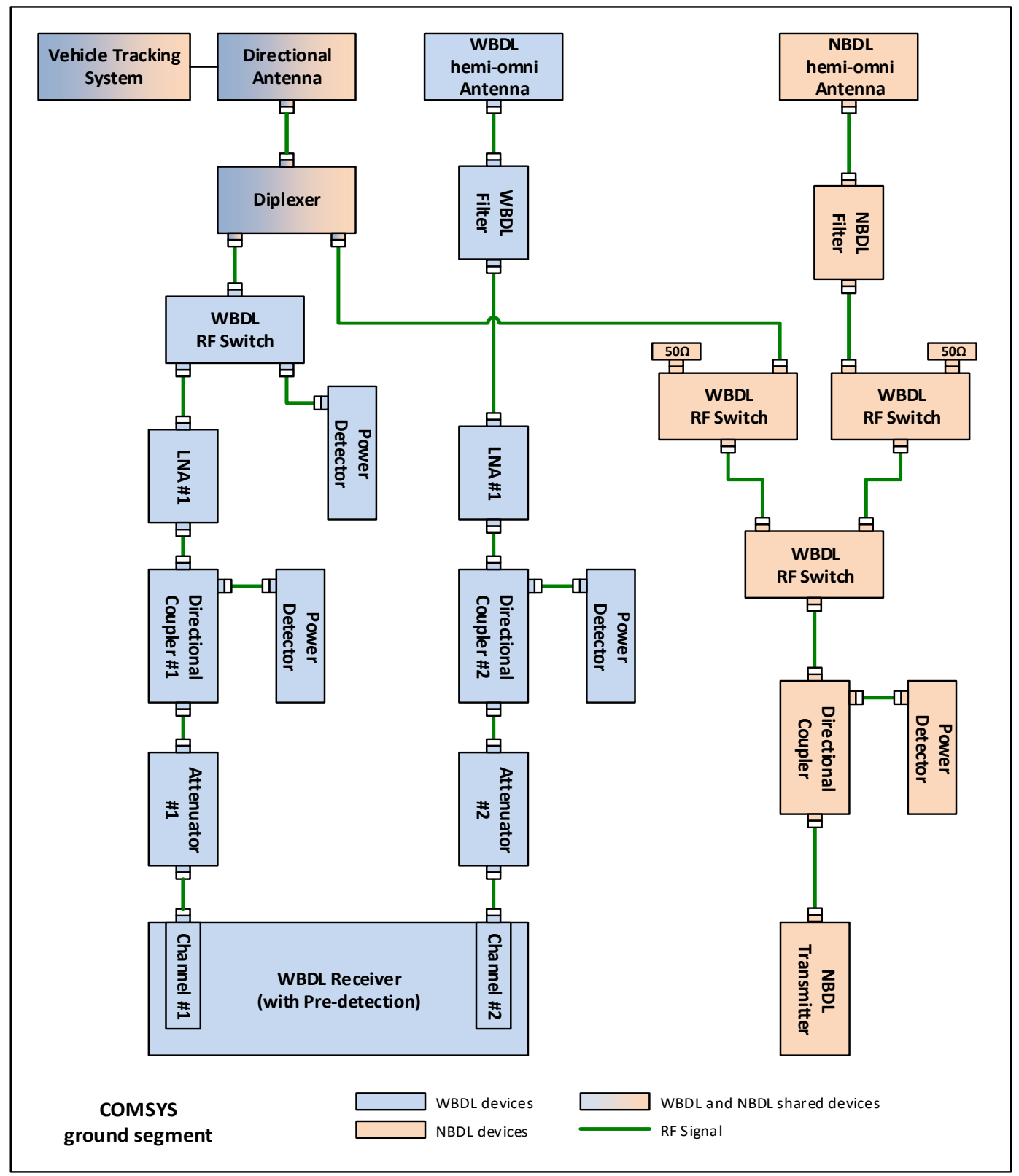

Figure 3. COMSYS Ground Segment Architecture 
The directional antenna is a parabolic antenna with a diameter of $1.2 \mathrm{~m}$. A tracking system, called Vehicle Tracking System (VTS), was in-house designed to properly point the parabolic antenna (see Figure 4) by basing on a mast with a two-degree of freedom mechanism, one for the rotation in the azimuth plane and one for the rotation in the elevation plane [9].

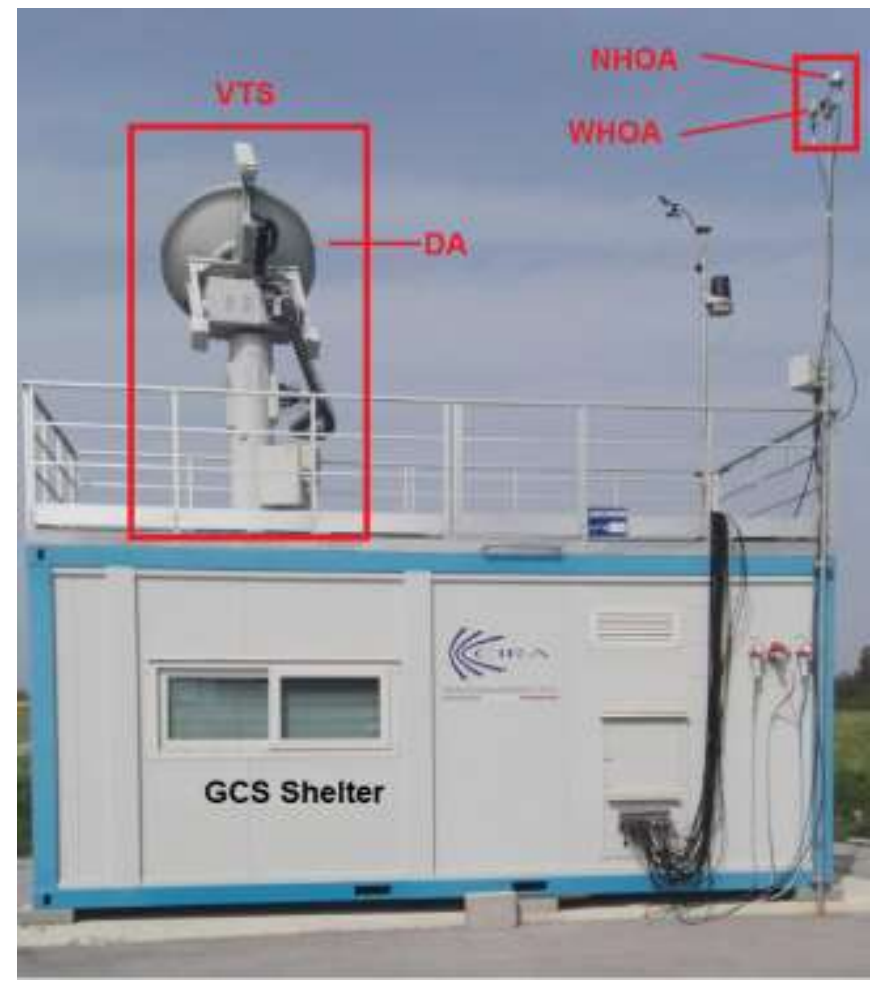

Figure 4. Ground Control Station with VTS, DA, WHOA and NHOA Antennas

With the exception of the motor drivers used for the azimuth and elevation rotations (which are provided with a programmable, embedded, PLC-based, low-level control system and integrated in the VTS) the overall VTS control system is integrated in the GCS shelter and operated by two software modules in-house developed. The first module is Windows-based and it is used to:

- display the antenna beam position and the status of the system,

- manually control the rotations and switch to the automatic control mode.

The second software module works on a real-time operating system and is used to:

- compute the set-point angles (azimuth and elevation) needed to control the VTS in automatic mode,

- transmit the set point angles to the VTS PLC-based low-level control system in both automatic and manual control modes.

The set-point angles are computed by basing on the aircraft GPS positions (received by the WBDL channel) and the GPS position of the ground station. To improve the availability of the system when a telemetry data loss occurs on the WBDL channel, the real-time software module is provided with an algorithm (based on an alpha-beta filter [5]) able to predict the aircraft position by basing on the last aircraft positions received by telemetry in the ground station. 


\section{External Interfaces}

COMSYS interfaces with the other devices of the on-board and ground segments by means of two Multiplexing/De-multiplexing Sub-systems (MDSs), integrated respectively in the aircraft and in the GCS. In the on-board segment, COMSYS:

- transmits to the GCS the multiplexed binary stream generated by the multiplexer of the On-board MDS (OMDS);

- receives the multiplexed binary stream generated by the multiplexer of the Ground MDS (GMDS) and sends it to the de-multiplexer of the OMDS.

Similar but complementary operations are carried out in the ground segment. By means of the MDSs, it is possible to convey in one RF link data coming from several data busses:

- an asymmetric board/ground Ethernet network (with a down-link throughput greater than the up-link throughput) used to transmit the out-the-window video, the set-up configuration and services data;

- four different point-point full-duplex asynchronous serial communication buses (RS-232); one of them is used for telemetry data in downlink and for telecommands in up-link.

MDSs are modular systems, so they can be simply upgraded to handle other kinds of communication buses (e.g.: serial synchronous buses, PCM channels), MIL-STD-1553 bus monitors or to exchange audio signal with the GCS.

\section{COMSYS Main Parameters}

In this section, the summary of the main COMSYS parameters is shown. In particular, for both WBDL and NBDL channels the following parameters are listed: Operating Frequency Range (OFR), frequency range where carrier (CFR) can be set, modulation, figure of merit $\left(\mathrm{G} / \mathrm{T}_{\mathrm{sys}}\right)$ of the receiving segment, minimum EIRP radiated by transmitting segment $^{2}, \mathrm{E}_{\mathrm{b}} / \mathrm{N}_{0}$ value to get a certain Bit Error Rate (BER).

Table 1. COMSYS Main Parameters

\begin{tabular}{|c|c|c|}
\hline \multirow[t]{2}{*}{ PARAMIETERS } & WBDL Channel & NBDL Channel \\
\hline & Values & Values \\
\hline OFR & $2360 \div 2400 \mathrm{MHz}$ & $2200 \div 2220 \mathrm{MHz}$ \\
\hline CFR & $2360 \div 2394.5 \mathrm{MHz}$ & $2200.5 \div 2220 \mathrm{MHz}$ \\
\hline Modulation & PCM - FM & PCM - FM \\
\hline MIN EIRP@Blade Antenna & $39.8 \mathrm{dBm}$ & - \\
\hline MIN EIRP@DA & - & $49.8 \mathrm{dBm}$ \\
\hline MINEIRP@NHOA & - & $25.5 \mathrm{dBm}$ \\
\hline G/Tsys with DA & $-11.8 \mathrm{~dB} / \mathrm{K}$ & - \\
\hline $\mathrm{G} / \mathrm{T}_{\text {sys }}$ with WHOA & $-34.5 \mathrm{~dB} / \mathrm{K}$ & - \\
\hline $\mathrm{G} / \mathrm{T}_{\text {sys }}$ with Blade Antenna & - & $-31.3 \mathrm{~dB} / \mathrm{K}$ \\
\hline $\mathrm{E}_{\mathrm{b}} / \mathrm{N}_{0} @ \mathrm{BER}=10^{-6}$ & $12.0 \mathrm{~dB}$ & $9.8 \mathrm{~dB}$ \\
\hline
\end{tabular}

The minimum EIRP for the on-board transmitting segment was evaluated considering the maximum attenuation between the WBDL transmitter and the blade antenna within the OFR and the minimum gain provided by the blade antenna. A minimum blade antenna

${ }^{2}$ EIRP and G/T $\mathrm{T}_{\text {sys }}$ are evaluated for each antenna in the architecture. 
gain of $-1 \mathrm{~dB}$ was considered in the link budget, to calculate the RF power $\left(\mathrm{P}_{\mathrm{r}}\right)$ received by the ground antennas and define a preliminary architecture of the WBDL channel (for the NBDL channel the method is the same). As above mentioned, the radiation pattern of the blade antenna installed under the aircraft fuselage was evaluated by an electromagnetic simulation tool. Then, according to:

- the power $\mathrm{P}_{\mathrm{r}}$,

- the angular spans of the blade antenna radiation pattern where the gain was larger than $-1 \mathrm{~dB}$,

- a constant maximum value for the fading margin (19dB),

- an aircraft roll angle varying in the range $\left[-40^{\circ}, 40^{\circ}\right]$,

- an aircraft pitch angle varying in the range $\left[-12^{\circ}, 12^{\circ}\right]$,

- a spatial region where the vehicle is permitted to fly, defined approximatively by an hemi-sphere with a $10 \mathrm{~km}$ radius,

the percentage of the space radio coverage evaluated in the above region was of $99.75 \%$, compatible with flight mission requirements.

$\mathrm{G} / \mathrm{T}_{\text {sys }}$ parameter was computed ([6], [7]) for all the COMSYS antennas considering the following worst cases: for the WHOA antenna, the minimum gain in the elevation range $\left[-5^{\circ}, 90^{\circ}\right]$ was assumed; for the DA antenna, a pointing error less than HPBW/2 (where HPBW is the Half Power Beam Width) and consequently a gain equal to the maximum mentioned into the datasheet $(24 \mathrm{dBi})$ reduced by $3 \mathrm{~dB}$ was assumed; for the blade antenna, the above mentioned minimum value $(-1 \mathrm{~dB})$ was assumed. To calculate the minimum EIRPs similar considerations were done.

$\mathrm{E}_{\mathrm{b}} / \mathrm{N}_{0}$ parameter values were defined measuring experimentally the behavior shown by the on-board and ground receivers.

\section{COMSYS Performance}

\subsection{Expected Performance}

By using the parameters shown in Table 1, the performance of COMSYS was analytically evaluated. In particular, the maximum radio coverage distance was computed for both WBDL and NBDL channels, for four different throughputs (bit rates) and four different fading margin values. The largest fading margin value $(19 \mathrm{~dB})$ was defined by basing on the free Irregular Terrain Model (ITM) with settings compatible with the operating area where COMSYS had to be used; the second value is the fading margin typically used in aeronautical projects where the link budgets for telemetry \& telecommands links are evaluated; the third and fourth value were considered for an operating context where fading phenomenon is mitigated or can be completely neglected: e.g.: aircraft high enough on the horizon and tracked by a directional antenna. Furthermore, maximum polarization mismatch between transmitting and receiving antennas, a system margin of $1 \mathrm{~dB}$ and a noise bandwidth numerically equal to the throughput were considered. To calculate the radio coverage distance, a carrier frequency equal to $2378 \mathrm{MHz}$ was used for the WBDL channel and a carrier frequency equal to $2210 \mathrm{MHz}$ for the NBDL channel. Analytical performance is shown in the following tables. 
Table 2. WBDL Channel Analytical Performance

\begin{tabular}{|c|c|c|}
\hline $\begin{array}{c}\text { Throughputs } \\
{[\mathrm{kbps}]}\end{array}$ & $\begin{array}{c}\text { Fading Margin } \\
{[\mathrm{dB}]}\end{array}$ & {$[\mathrm{km}]$} \\
\hline 500 & 19 & 49.2 \\
\hline 500 & 10 & 138.6 \\
\hline 500 & 6 & 219.7 \\
\hline 500 & 0 & 438.4 \\
\hline 1700 & 19 & 26.7 \\
\hline 1700 & 10 & 75.2 \\
\hline 1700 & 6 & 119.2 \\
\hline 1700 & 0 & 237.8 \\
\hline 6000 & 19 & 14.2 \\
\hline 6000 & 10 & 40.0 \\
\hline 6000 & 6 & 63.4 \\
\hline 6000 & 0 & 126.6 \\
\hline 12000 & 19 & 10.0 \\
\hline 12000 & 10 & 28.3 \\
\hline 12000 & 6 & 44.8 \\
\hline 12000 & 0 & 89.5 \\
\hline
\end{tabular}

Table 3. NBDL Channel Analytical Performance

\begin{tabular}{|c|c|c|}
\hline Throughputs & Fading Margin & Maximum Distance \\
\hline$[\mathrm{kbps}]$ & {$[\mathrm{dB}]$} & {$[\mathrm{km}]$} \\
\hline 350 & 19 & 27.3 \\
\hline 350 & 10 & 76.9 \\
\hline 350 & 6 & 121.9 \\
\hline 350 & 0 & 243.3 \\
\hline 700 & 19 & 19.3 \\
\hline 700 & 10 & 54.4 \\
\hline 700 & 6 & 86.2 \\
\hline 700 & 0 & 172.0 \\
\hline 1000 & 19 & 16.1 \\
\hline 1000 & 10 & 45.5 \\
\hline 1000 & 6 & 72.1 \\
\hline 1000 & 0 & 143.9 \\
\hline 1700 & 19 & 12.4 \\
\hline 1700 & 10 & 34.9 \\
\hline 1700 & 6 & 55.3 \\
\hline 1700 & 0 & 110.4 \\
\hline
\end{tabular}

\subsection{Experimentally Evaluated Performance}

COMSYS experimental performance was evaluated taking advantage of the flight missions carried out for other projects. The missions were carried out taking off from Capua Airport "Oreste Salomone" with the following scenario: 
- the red shaded area (see Figure 5) is the segregated area where flight missions had to be executed when FLARE was remotely or automatically piloted; when FLARE was piloted by the on-board pilot, it could fly outside the segregated area as well;

- GCS was located at north of the airport (see Figure 6), near an hangar which could preclude or affect the LOS condition whenever the aircraft flew into the area delimited by the two blue lines shown in Figure 5 and Figure 6;

- a throughput of $1700 \mathrm{kbps}$ was selected for the down-link channel, including an asynchronous RS232 signal (with a baud rate of $115.2 \mathrm{kbps}$ ) dedicated to telemetry data packets and an Ethernet network down-link (with a throughput of $1400 \mathrm{kbps}$ ) dedicated to the out-the-window video and handshakes between the on-board video encoder and the ground video decoder;

- a throughput of 350kbps was selected for the up-link channel, including an asynchronous RS232 signal (with a baud rate of $115.2 \mathrm{kbps}$ ) dedicated to telecommand data packets, an asynchronous RS232 signal (with a baud rate of 9.6kbps) dedicated to the GPS differential correction, and an Ethernet network up-link (with a throughput of $128 \mathrm{kbps}$ ) dedicated to the handshake between the on-board video encoder and the ground video decoder.

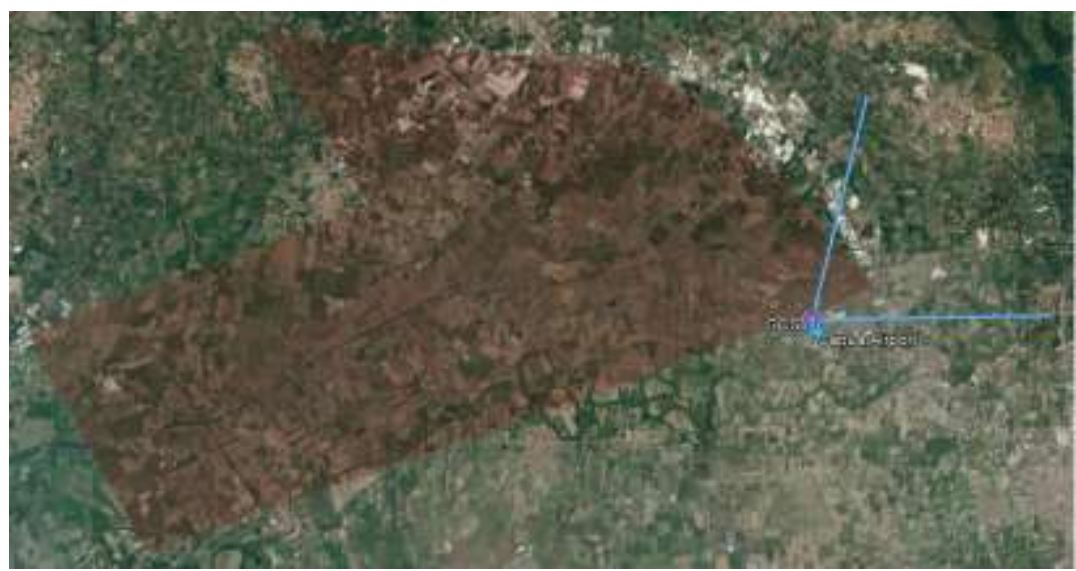

Figure 5. Shaded Red Color Represents the Segregated Area for the Flight Missions

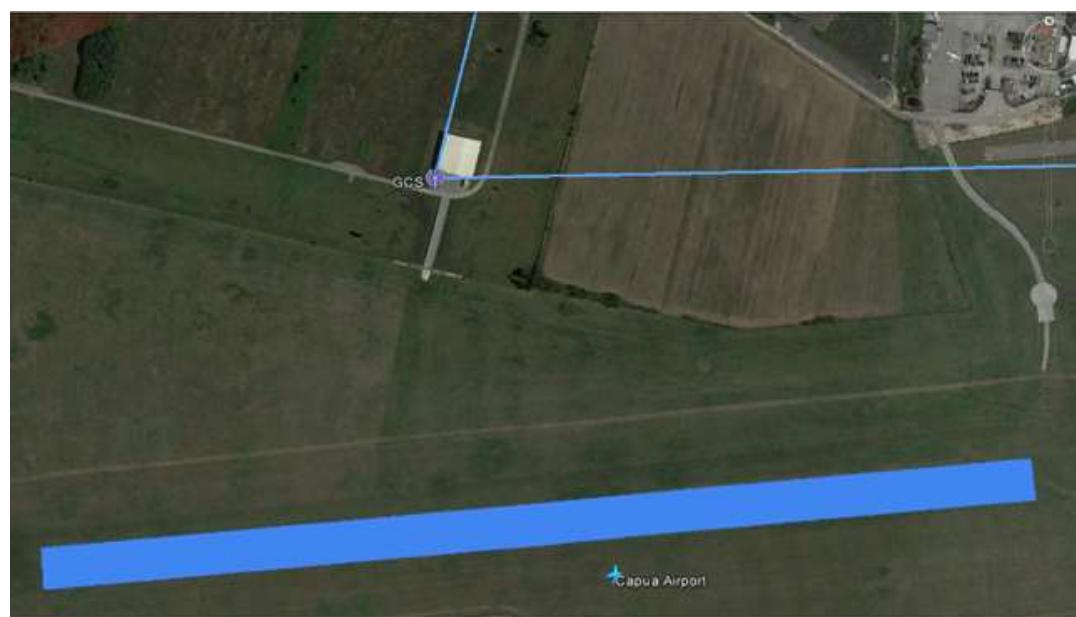

Figure 6. GCS Position Respect to the Capua Airport "Oreste Salomone" Runway 
During the flight missions telemetry data packets and command data packets were transmitted each with a frequency of $10 \mathrm{~Hz}$. Data packets were encapsulated into frames (together with the other transmitted data) using a CCSDS compliant protocol implemented by OMDS in the on-board segment and by GMDS in the ground segment. The frames were transmitted in synchronous mode generating a continuous bitstream. The used protocol was transparent to the user so it would be difficult to evaluate the actual bit error rate by means of a bitstream analysis. To evaluate the quality of service (QoS) related to up and down links, lost packet rates for both channels were computed.

For the down-link, the evaluation of the Packet Loss Ratio (PLR) ${ }^{3}$ was based on data recorded during 16 flight missions. For each flight, the results were separated in two different phases: the taxing phase and the flight phase (the latter includes the take-off). Results are shown in Table 4 and Table 5. In particular, the following parameters were evaluated: quantity of lost packets, Packet Loss Ratio, length (in terms of packets) of the longest errors burst, quantity of the errors bursts (QoEB) having a length greater than or equal to 10 packets (i.e. at least one-second-long bursts).

Table 4. Taxing Phase, Performance Analysis for WBDL Channel

\begin{tabular}{|c|c|c|c|c|c|}
\hline \multirow{3}{*}{ Mission } & \multicolumn{5}{|c|}{ Taxing Phase } \\
\hline & \multirow{2}{*}{$\begin{array}{c}\text { Total } \\
\text { Transmitted } \\
\text { Packets }\end{array}$} & \multirow[b]{2}{*}{ Lost Packets } & \multirow{2}{*}{$\begin{array}{c}\text { Lost } \\
\text { Packets \% }\end{array}$} & \multicolumn{2}{|c|}{ Error Bursts } \\
\hline & & & & Max length & $\begin{array}{c}\text { QoEB }>10 \\
\text { packets }\end{array}$ \\
\hline$\overline{\# 1}$ & 4020 & 31 & $0.8 \%$ & 7 & 0 \\
\hline$\# 2$ & 2701 & 10 & $0.4 \%$ & 4 & 0 \\
\hline \#3 & 3500 & 35 & $1.0 \%$ & 4 & 0 \\
\hline$\overline{\# 4}$ & 1500 & 46 & $3.1 \%$ & 17 & 2 \\
\hline$\# 5$ & 2601 & 18 & $0.7 \%$ & 3 & 0 \\
\hline \#6 & 3000 & 16 & $0.5 \%$ & 5 & 0 \\
\hline$\# 7$ & 2081 & 35 & $1.7 \%$ & 6 & 0 \\
\hline$\# 8$ & 1990 & 13 & $0.7 \%$ & 2 & 0 \\
\hline$\# 9$ & 1741 & 31 & $1.8 \%$ & 8 & 0 \\
\hline$\# 10$ & 2441 & 0 & $0.0 \%$ & 0 & 0 \\
\hline$\# 11$ & 3299 & 55 & $1.7 \%$ & 11 & 1 \\
\hline$\# 12$ & 1880 & 59 & $3.1 \%$ & 23 & 1 \\
\hline$\# 13$ & 4500 & 44 & $1.0 \%$ & 4 & 0 \\
\hline$\# 14$ & 3100 & 41 & $1.3 \%$ & 15 & 1 \\
\hline$\# 15$ & 3350 & 69 & $2.1 \%$ & 16 & 2 \\
\hline$\# 16$ & 3000 & 60 & $2.0 \%$ & 12 & 1 \\
\hline Total & 44704 & 563 & $1.3 \%$ & 23 & 8 \\
\hline
\end{tabular}

Table 5. Flight Phase, Performance Analysis for WBDL Channel

\begin{tabular}{|c|c|c|c|c|c|}
\hline \multirow{3}{*}{ Mission } & \multicolumn{5}{|c|}{ Flight Phase } \\
\hline & \multirow{2}{*}{$\begin{array}{c}\text { Total } \\
\text { Transmitted } \\
\text { Packets }\end{array}$} & \multirow[b]{2}{*}{ Lost Packets } & \multirow{2}{*}{$\begin{array}{c}\text { Lost } \\
\text { Packets \% }\end{array}$} & \multicolumn{2}{|c|}{ Error Bursts } \\
\hline & & & & Max length & $\begin{array}{c}\text { QoEB }>10 \\
\text { packets }\end{array}$ \\
\hline$\# 1$ & 17961 & 88 & $0.5 \%$ & 31 & 1 \\
\hline$\# 2$ & 15660 & 33 & $0.2 \%$ & 8 & 0 \\
\hline
\end{tabular}

\footnotetext{
${ }^{3}$ The Packet Loss Ratio is defined as the number of the lost packets over the number of transmitted packets.
} 


\begin{tabular}{|c|c|c|c|c|c|}
\hline$\# 3$ & 1550 & 2 & $0.1 \%$ & 2 & 0 \\
\hline$\# 4$ & 28581 & 250 & $0.9 \%$ & 53 & 5 \\
\hline$\# 5$ & 19760 & 13 & $0.1 \%$ & 2 & 0 \\
\hline$\# 6$ & 28681 & 12 & $0.0 \%$ & 2 & 0 \\
\hline$\# 7$ & 19910 & 227 & $1.1 \%$ & 49 & 6 \\
\hline$\# 8$ & 26641 & 63 & $0.2 \%$ & 18 & 2 \\
\hline$\# 9$ & 18431 & 18 & $0.1 \%$ & 2 & 0 \\
\hline$\# 10$ & 7520 & 4 & $0.1 \%$ & 1 & 0 \\
\hline$\# 11$ & 19551 & 17 & $0.1 \%$ & 2 & 0 \\
\hline$\# 12$ & 18071 & 16 & $0.1 \%$ & 4 & 0 \\
\hline$\# 13$ & 29851 & 56 & $0.2 \%$ & 18 & 1 \\
\hline$\# 14$ & 22221 & 10 & $0.0 \%$ & 1 & 0 \\
\hline$\# 15$ & 8180 & 3 & $0.0 \%$ & 2 & 0 \\
\hline$\# 16$ & 17360 & 11 & $0.1 \%$ & 2 & 0 \\
\hline Total & $\mathbf{2 9 9 9 2 9}$ & $\mathbf{8 2 3}$ & $\mathbf{0 . 3 \%}$ & $\mathbf{5 3}$ & $\mathbf{1 5}$ \\
\hline
\end{tabular}

For the up-link, the evaluation of the packet loss ratio was based only on 12 out of 16 missions examined for the down-link. In the up-link case, data were available only for the flight phase (notice that the take-off is included only in three flight missions, but it seem to have no effects on the performance). Results are shown in Table 6.

Table 6. Flight Phase, Performance Analysis for NBDL Channel

\begin{tabular}{|c|c|c|c|c|c|}
\hline \multirow{2}{*}{ Mission } & \multicolumn{5}{|c|}{ Flight Phase } \\
\cline { 5 - 6 } & \multirow{2}{*}{$\begin{array}{c}\text { Total } \\
\text { Pransmitted }\end{array}$} & \multirow{2}{*}{ Lost Packets } & \multirow{2}{*}{$\begin{array}{c}\text { Lost } \\
\text { Packets \% }\end{array}$} & Max length & $\begin{array}{c}\text { QoEB }>\text { 10 } \\
\text { packets }\end{array}$ \\
\cline { 5 - 6 }$\# 1$ & 17960 & 92 & $0.5 \%$ & 9 & 0 \\
\hline$\# 2$ & 15660 & 98 & $0.6 \%$ & 8 & 0 \\
\hline$\# 4$ & 16054 & 44 & $0.3 \%$ & 4 & 0 \\
\hline$\# 5$ & 19759 & 28 & $0.1 \%$ & 6 & 0 \\
\hline$\# 6$ & 28679 & 87 & $0.3 \%$ & 50 & 1 \\
\hline$\# 7$ & 19909 & 139 & $0.7 \%$ & 8 & 0 \\
\hline$\# 9$ & 16793 & 11 & $0.1 \%$ & 4 & 0 \\
\hline$\# 10$ & 4832 & 0 & $0.0 \%$ & 0 & 0 \\
\hline$\# 13$ & 25126 & 63 & $0.3 \%$ & 46 & 1 \\
\hline$\# 14$ & 19419 & 2 & $0.0 \%$ & 1 & 0 \\
\hline$\# 15$ & 5233 & 0 & $0.0 \%$ & 0 & 0 \\
\hline$\# 16$ & 12479 & 0 & $0.0 \%$ & 0 & 0 \\
\hline Total & $\mathbf{2 0 1 9 0 3}$ & $\mathbf{5 6 4}$ & $\mathbf{0 . 3 \%}$ & $\mathbf{5 0}$ & $\mathbf{2}$ \\
\hline
\end{tabular}

The total packet loss ratio for the flight phase is similar (less than $\sim 0.3 \%$ ) for up and down links. This result is consistent with the analytical results, because with the selected throughputs (1700kbps for WBDL and to 350kbps for NBDL) the two channels have similar radio coverage (see Table 2 and Table 3, i.e. similar performance. Even though the two channels occupied different frequency bandwidths (due to different throughputs), the prevalent use of the directional antenna during the flight phase let the frequency selective fading be negligible. 
For the taxing phase, the packet loss ratio, available only for the down-link, is about five times greater than the packet loss ratio in the flight phase. The performance drop is due to frequency selective fading generated by the ground reflections (the blade antenna is located in the bottom of the fuselage), which has a non-negligible impact on the quality of the RF signal received by the ground station.

By referring to the error bursts detected in the down-link channel, we had:

- 8 bursts longer than 1 second over $\sim 4.500$ seconds of data transmission during the taxing phase, with 23 lost packets in the longest burst;

- 15 bursts longer than 1 second over 30.000 seconds of data transmission during the flight phase, with 53 lost packets in the longest burst.

In the taxing phase, the 8 bursts are still due to the frequency selective fading generated by the ground reflections. In the flight phase, all the bursts longer than 1 second occurred when the aircraft crossed the area overshadowed by the hangar (positioned behind the GCS), with the exception of a unique burst related to a still unexplained sudden drop of the received power.

For the up-link channel we had only 2 bursts longer than 1 second over $\sim 20.000$ seconds of data transmission during the flight phase, with 50 lost packets in the longest burst (the cause of these two bursts requires further analysis).

In Figure 7, lost packets vs. flight time (for the down-link case) is shown for a typical mission (flight mission number \#6), as well as the received power @ WBDL receiver channels, the altitude and roll \& pitch angles of the aircraft.
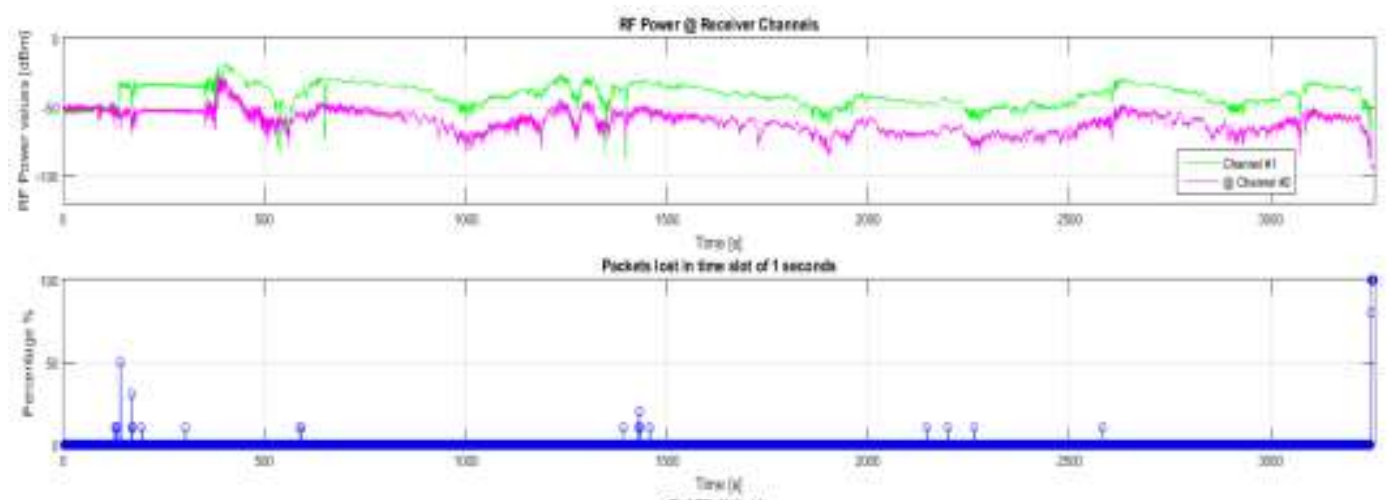

Fuare allash

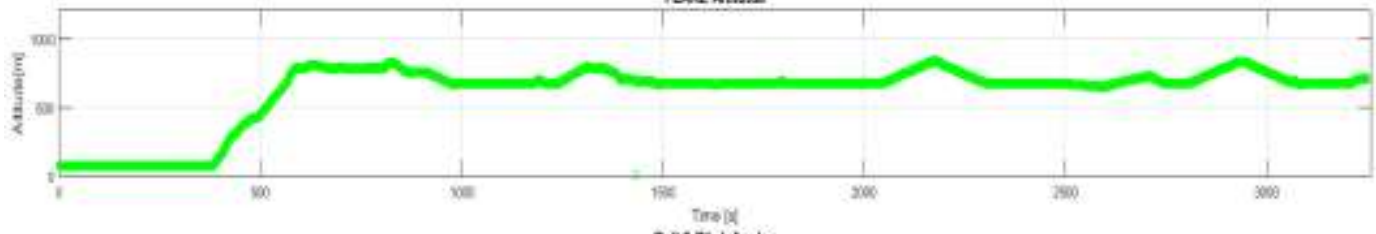

axtis patstinges

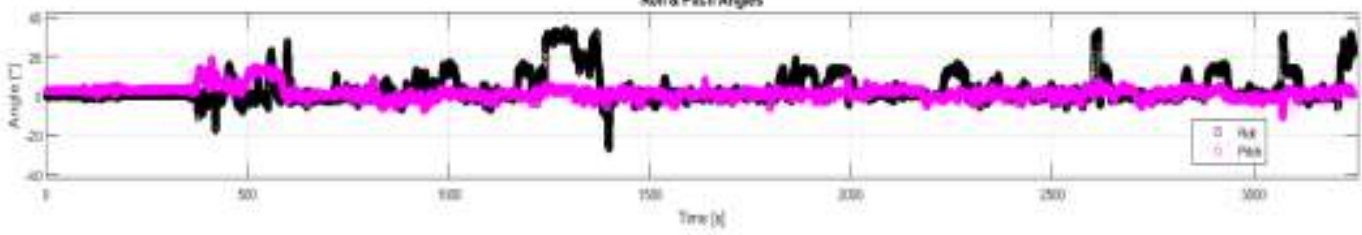

Figure 7. Data Analysis for the Flight Mission Number \#6

\subsection{Experimental Latency Time}

COMMSYS provides low latency end-to-end data transfer from the multiplexing unit on one end to the de-multiplexing unit on the other end. In particular, two configurations were tested in a "loop-back" type setup (see Figure 8). Considering negligible the delay 
introduced by the radio channel (i.e., less than $0.1 \mathrm{~ms}$ for a distance between the aircraft and the ground control station of about $30 \mathrm{~km}$ ), the maximum round-trip latency time (i.e., the sum of T1 time and T2 time shown in Figure 8) is equal respectively to $\sim 6 \mathrm{~ms}$ and to $33 \mathrm{~ms}$ for the two configurations. Consequently, the time delay per route is respectively equal to $\sim 3 \mathrm{~ms}$ and $\sim 16.5 \mathrm{~ms}$.

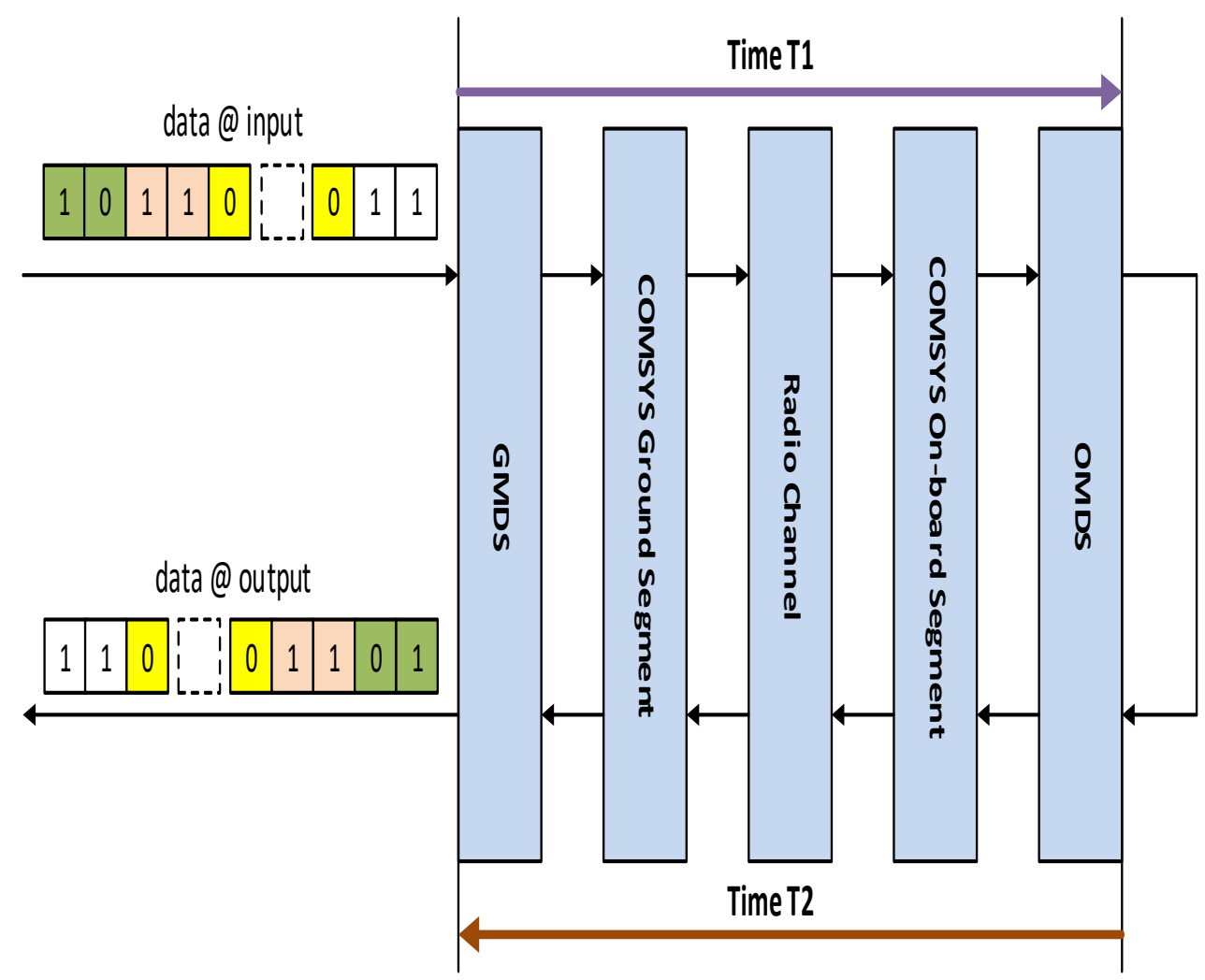

Figure 8. COMSYS Latency Time Measurement Sketch

\section{Conclusions}

In this paper the architecture and the experimental performance of the COMSYS datalink system, used as Command and Control datalink for an RPAS based on an OPA, have been described.

COMSYS is a full duplex communication system working in the $\mathrm{S}$ band, made up by two different omnidirectional simplex communication channels, the first one for the uplink and the second one for the down-link. The down-link was designed to support throughput up to $12 \mathrm{Mbps}$ with a radio coverage of at least $10 \mathrm{~km}$; the up-link channel is designed to support throughput up to $1 \mathrm{Mbps}$ with a radio coverage of at least $16 \mathrm{~km}$. COMSYS interfaces with two Multiplexing/Demultiplexing Systems to transfer different kind of data from the aircraft to the ground station and vice versa with a very low latency time.

Experimental performances confirmed the reliability of the system showing a packet loss ratio less than $0.3 \%$ both for down-link and up-link during the flight phase. For the taxing phase experimental results are available only for the down-link; in this case we have a packet loss ratio less than $1.3 \%$. In all the analyzed phases the number of lost packet bursts is negligible compared to the total transmitted data. 


\section{References}

[1] L. Vecchione, P. De Matteis, A. Rispoli, L. Pellone, U. Mercurio, M. Di Donato, A. Pagano, M. Inverno, "FLARE: An OPA for technology validation used at the Italian Aerospace Research Center", Proceedings of the Society of Flight Test Engineer, 47th Annual International Symposium, Wichita, Kansas, USA (2016), August 8-11.

[2] A. Rispoli, U. Mercurio, L. Vecchione, P. de Matteis, F. Fusco, "Exploitation of an OPA platform for inflight validation of RPAS technologies", Proceedings of the AIAA Aviation Forum - Flight Test Conference, Denver, CO, USA (2017) June 5-9.

[3] R. Rocchio, F. Corraro, U. Ciniglio, L. Garbarino, F. Fusco, V. Castrillo, A. Rispoli, "Flight Testing Avionics of an Optionally Piloted Vehicle for UAS Integration in the Civil Airspace" Proceedings of the 36th Digital Avionics Systems Conference, St. Petersburg, Florida, USA (2017), September 17-21.

[4] FAA order 8130.34, "Airworthiness Certification of Unmanned Aircraft Systems and Optionally Piloted Aircraft", Appendix F, Nov. 28 ${ }^{\text {th }}$, (2011).

[5] J. E. Gray and W. J. Murray, "A Derivation of an Analytic Expression for the Tracking Index for the Alpha-Beta Filter", IEEE Transactions on Aerospace and Electronic Systems, vol. 29, issue 3, (1993), pp. 1064-1065.

[6] L.W. Couch II, "Digital And Analog Communication Systems", 5th edition, Pearson, New Jersey, (1996), pp. 560-575.

[7] F. Carden, R. Jedlicka, R. Henry, Telemetry Systems Engineering, Artech House, Norwood - MA 02062, (2002), pp. 139-165.

[8] V. U. Castrillo, F. Fusco, M. Inverno, R. V. Montaquila, G. Buzzo, L. Garbarino, G. Di Capua, A. Rispoli, L. Vecchione, "Ground Segment for Flight Tests of an Optionally Piloted Aircraft of the Italian Aerospace Research Center", Proceedings of the European Test and Telemetry Conference, Toulouse, France, (2017) June 13-15.

[9] F. Fusco, M. Inverno, V. Castrillo, R. Montaquila, G. Buzzo, S. Immediata, E. Memoli, "Design and validation of a Vehicle Tracking System based on GNSS data and supporting the communications with the CIRA's Optionally Piloted Aircraft", Proceedings of the 24th International Conference of Italian Association of Aeronautics and Astronautics, Palermo - Enna, Italy, (2017), September 18-22.

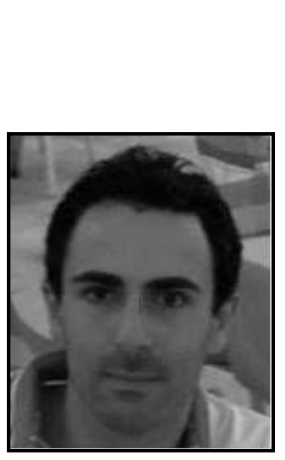

\section{Authors}

Vittorio Ugo Castrillo. He received the M.S. degree with honors in Electronic Engineering from the University of Naples "Federico II" (Italy) in 2003. He joined the Electronics and Communications group of the Italian Aerospace Research Centre (C.I.R.A.) in 2004. In the first years of activity he was involved in the design and integration of data acquisition systems and in the design and development of software for microcontroller-based applications. Afterwards he has worked on the design and integration of communication systems for telemetry and control data-link for unmanned aerial and space vehicles. Since 2011 his activities have been focused also on the design of algorithms for base-band digital signal processing for communication systems, with their implementation on FPGA-based hardware platforms. Recently, until the end of 2016, he was project manager for the design of an innovative architecture for the implementation of a beam steering controller of a phased array.

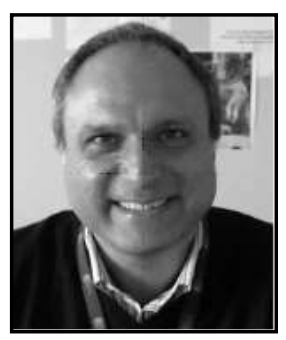

Francesco Fusco. He took his graduation with honors in Physics in 1988. He worked as system engineer at the IBM Telecommunication Development Center for about one year. Since the end of 1989, he has been working for the Italian Aerospace Research Center (CIRA) and in 1993 he became responsible of the "Data Acquisition System" unit where he was involved in the design and integration of the Measurement \& Data Acquisition Systems of all the CIRA large facilities: Plasma Wind Tunnel, Icing Wind 
Tunnel, crash test facility for aeronautical structures, Transonic Wind Tunnel. For two years he was responsible of the "System Engineering and Integration" unit whose mission was the design, manufacturing and integration of electronic and mechanical equipment to support inflight and on-ground experimental tests for Unmanned Aerial Vehicle (UAV) and Unmanned Space Vehicle (USV) programs. From the beginning of 2011 up to the end of 2014 he was responsible of the "Electronic Systems \& Instrumentation" unit coordinating three subunits: "Embedded systems \& communications", "Payload sensors \& HMI" and "Meteo systems and instrumentation". Since the beginning of 2015 he has been working as responsible of the "Electronics and Communications" unit.

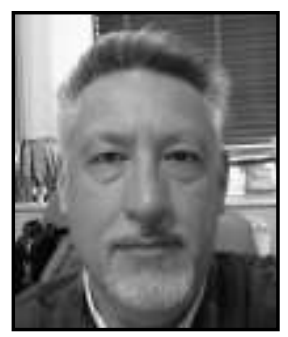

Michele Inverno. He graduated in Electronics Engineering in 1992. He worked as electronic hardware designer at the Olivetti Research in the Hardware Development Laboratory for about one year. Since the 1994 he has been working for CIRA as hardware and software engineer in the "Data Acquisition and Instrumentation" unit (currently "Electronics and Microelectronics" unit), he was involved in national and international projects mainly concerning material characterization and data acquisition systems design. In 1998 he collaborated with INFM (National Institute for Physics of Material) for new magnetostrictive sensors development. He was involved in the system design of several on-board electronic subsystems for data handling, electrical power distribution, RF datalinks and, more in general, for the integration and test of the overall experimental setups - including flight and ground segments - of the CIRA experimental flying platforms for flight tests. In 2005 he was system engineer of the USV (Unmanned Space Vehicle) Passenger EXperiment and in 2006 he was manager of the USV AIT/AIV. From December 2010, he was the responsible of the Embedded Systems and Communications unit. Since 2015 he is the responsible of Electronic and Microelectronic unit. 
International Journal of Future Generation Communication and Networking Vol. 10, No. 11 (2017) 